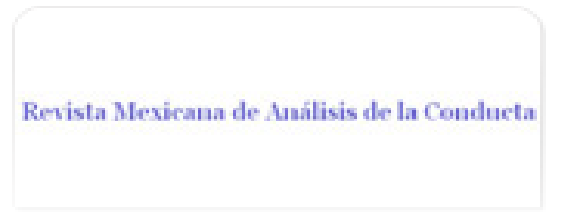

Revista Mexicana de Análisis de la Conducta ISSN: 0185-4534

editora@rmac-mx.org

Sociedad Mexicana de Análisis de la Conducta México

Serrano, Mario; Camacho, Isaac; Carpio, Claudio Intervalos entre ensayos de distinta duración en igualación de la muestra demorada Revista Mexicana de Análisis de la Conducta, vol. 32, núm. 1, junio, 2006, pp. 1-12

Sociedad Mexicana de Análisis de la Conducta Guadalajara, México

Disponible en: http://www.redalyc.org/articulo.oa?id=59332101

- Cómo citar el artículo

- Número completo

- Más información del artículo

- Página de la revista en redalyc.org

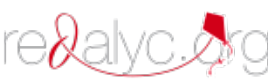

Sistema de Información Científica Red de Revistas Científicas de América Latina, el Caribe, España y Portugal Proyecto académico sin fines de lucro, desarrollado bajo la iniciativa de acceso abierto 


\title{
INTERVALOS ENTRE ENSAYOS DE DISTINTA DURACIÓN EN IGUALACIÓN DE LA MUESTRA DEMORADA ${ }^{1,2}$
}

\author{
DIFFERENT LENGTH INTERTRIAL INTERVALS \\ IN DELAYED MATCHING-TO-SAMPLE \\ MARIO SERRANO, ISAAC CAMACHO Y CLAUDIO CARPIO \\ UNIVERSIDAD NACIONAL AUTÓNOMA DE MÉXICO \\ FACULTAD DE ESTUDIOS SUPERIORES IZTACALA
}

\begin{abstract}
RESUMEN
Dos grupos de pichones fueron expuestos a un procedimiento sucesivo de igualación de la muestra demorada. Para un grupo los estímulos de muestra se correlacionaron diferencialmente con intervalos entre ensayos de distinta duración, mientras para el otro grupo la duración del intervalo entre ensayos era la misma dado uno u otro estímulo de muestra. Para ambos grupos el índice de precisión disminuyó al incrementar la duración del intervalo de demora, sin embargo, este efecto fue menor para el grupo expuesto al procedimiento con intervalos entre ensayos de distinta duración. Adicionalmente, sólo para este grupo se observaron diferentes tasas de respuesta ante los estímulos de muestra. Los resultados se discuten en relación con estudios comparables, destacando las virtudes de una aproximación paramétrica en el análisis experimental del comportamiento en situaciones de discriminación condicional.
\end{abstract}

1. La correspondencia relacionada con este trabajo puede dirigirse al último autor a Universidad Nacional Autónoma de México, Facultad de Estudios Superiores Iztacala. Av. De los Barrios No. 1, Los Reyes Iztacala. Tlalnepantla, Estado de México. C. P. 54090. Correo electrónico: carpio@servidor.unam.mx

2. El presente trabajo recibió apoyo parcial del financiamiento PAPIIT-IN301203 de la Dirección General de Asuntos del Personal Académico, otorgado al último autor. Los autores agradecen a los revisores por sus atinadas recomendaciones para mejorar el manuscrito, así como a los doctores Emilio Ribes y François Tonneau y los miembros del Grupo T de Investigación Interconductual, por sus comentarios a una versión previa. 
Palabras clave: Igualación de la muestra demorada, intervalo entre ensayos, responder diferencial a los estímulos de muestra, pichones.

\section{ABSTRACT}

Two groups of pigeons were exposed to a successive delayed matching-tosample procedure. For one group sample stimuli were differentially correlated with different length intertrial intervals, whereas for the other group the length of the intertrial interval was the same given either sample stimuli. For both groups precision index decreased as delay interval length increased; however, this effect was lesser for the group exposed to the procedure with different length intertrial intervals. Additionally, for this group only different local response rates at sample stimuli was observed. Results are discussed in relation to similar experiments, highlight the virtues of a parametric approximation to the experimental analysis of behavior in conditional discrimination situations.

Keywords: Delayed matching-to-sample, intertrial interval, differential sample responding, pigeons.

En los procedimientos de igualación de la muestra demorada se introduce un intervalo de demora (ID) entre la terminación del estímulo de muestra (EM) y el inicio de los estímulos de comparación (ECOs)(Berryman, Cumming \& Nevin, 1963). La precisión de la ejecución en este tipo de procedimientos es afectada por distintos parámetros temporales. Se sabe, por ejemplo, que el índice de discriminación generalmente disminuye conforme se incrementa la duración del ID, pero que aumenta al incrementar la duración del intervalo entre ensayos (IEE) y/o la duración de los EMs (e.g., Nelson \& Wasserman, 1978).

Aunque en la mayoría de los estudios que evaluaron los efectos de la duración de los EMs se programaron duraciones intrasesión equivalentes entre los estímulos, experimentos recientes han evaluado los efectos de programar diferentes duraciones de los EMs al interior de la sesión experimental. Hartl, Dougherty y Wixted (1996, Experimento 2), por ejemplo, expusieron a varios pichones a un procedimiento de igualación de la muestra demorada en el que los EMs se presentaron durante 5 y $15 \mathrm{~s}$. En dos condiciones cada EM se correlacionó con una duración específica, mientras en una condición intermedia la duración de ambos EMs se determinó en forma aleatoria. Los autores observaron que aunque en todas las condiciones el porcentaje de respuestas correctas disminuyó conforme se incrementó la duración del ID, el efecto fue menor en las condiciones correlacionadas que en la condición 
no correlacionada. El mismo resultado fue reportado por Urcuioli, DeMarse y Lionello (1999, Experimento 2), utilizando un diseño de grupos y un procedimiento de igualación de la muestra demorada con cuatro EMs y dos ECOs (i.e., procedimiento "muchos a uno").

El efecto de diferenciar los arreglos de estímulo en procedimientos de igualación de la muestra demorada no se limita a la duración de los EMs. Estudios previos han reportado resultados similares al correlacionar cada EM con distintas probabilidades de reforzamiento (e.g., DeLong \& Wasserman, 1981) y con diferentes duraciones del ID (e.g., Honig, 1987). No obstante, hasta el momento se desconoce si el efecto puede extenderse al IEE. El presente experimento intentó subsanar esta limitación. Específicamente, evaluó los efectos de correlacionar los EMs con IEEs de distinta duración, sobre el índice de precisión en un procedimiento sucesivo de igualación de la muestra demorada.

\section{MÉTODO}

\section{Sujetos}

Se utilizaron seis pichones de la raza Columba livia, experimentalmente ingenuos, mantenidos al $75 \%$ de su peso en alimentación libre mediante restricción de alimento. Los sujetos tuvieron acceso libre al agua en sus jaulas hogar a lo largo de todo el experimento.

\section{Aparatos}

Se utilizó una cámara de condicionamiento operante marca Coulbourn Instruments $®$ modelo E10-10 $(31 \mathrm{~cm} \times 30.5 \mathrm{~cm} \times 25.5 \mathrm{~cm})$. En el panel frontal de la cámara y a $21 \mathrm{~cm}$ del piso de rejilla, se colocó una tecla de respuesta translúcida de $2.5 \mathrm{~cm}$ de diámetro, en la que se retroproyectaron luces de colores y figuras geométricas. Detrás de la tecla de respuesta, se colocó un microinterruptor que requirió una fuerza de $20 \mathrm{gr}$ de peso muerto para cerrarse. La apertura para el dispensador de alimento se colocó a $17 \mathrm{~cm}$ debajo de la tecla de respuesta y en cada presentación del reforzador se iluminó con una luz blanca de $5 \mathrm{w}$. Para eliminar los ruidos ajenos al experimento, la cámara experimental se colocó dentro de un cubículo de aislamiento acústico marca Coulbourn Instruments y se presentó ruido blanco constante. Para la programación y registro de los eventos, se utilizó un equipo de cómputo estándar provisto de una interfase y software MED ${ }^{\circledR}$. 


\section{Procedimiento}

Las sesiones experimentales se llevaron a cabo todos los días de la semana, colocando a los sujetos en la cámara experimental siempre en el mismo orden y horario. Después del establecimiento de la respuesta de picar la tecla mediante un procedimiento de automoldeamiento (Brown \& Jenkins, 1968), los sujetos fueron divididos de forma aleatoria en dos grupos de tres sujetos cada uno y expuestos a las siguientes condiciones experimentales:

Igualación de la muestra demora cero: Cada ensayo comenzó con la iluminación de la tecla de respuesta mediante una luz roja (EM1) o verde (EM2) durante $5 \mathrm{~s}$, al término de los cuales se presentó un círculo (ECO1) o un triángulo (ECO2) durante $3 \mathrm{~s}$ adicionales. Las combinaciones EM1-ECO1 y EM2-ECO2 se designaron como ensayos positivos. Las combinaciones EM1-ECO2 y EM2-ECO1, se designaron como ensayos negativos. Los ensayos positivos en los que ocurrió al menos una respuesta ante el ECO, fueron seguidos de $3 \mathrm{~s}$ de acceso al comedero y se consideraron como aciertos por comisión. En los ensayos negativos, las respuestas emitidas ante el ECO no tuvieron consecuencias programadas y se consideraron como errores por comisión. Los ensayos positivos en los que no se emitió respuesta ante el ECO, se consideraron como errores por omisión y el reforzador no se presentó. Los ensayos negativos en los que no se emitió respuesta ante el ECO, se consideraron como aciertos por omisión y tampoco tuvieron consecuencias.

Los sujetos S1, S2 y S3 conformaron el Grupo 1. Para este grupo, los ensayos positivos y negativos que iniciaron con el EM1 se correlacionaron con un IEE de $30 \mathrm{~s}$, mientras los ensayos positivos y negativos que iniciaron con el EM2 se correlacionaron con un IEE de $5 \mathrm{~s}$. Los sujetos S4, S5 y S6 conformaron el Grupo 2. Para este grupo se programó un IEE de 17.5 s dado cualquiera de los EMs, tanto en los ensayos positivos como en los negativos. Durante el IEE, la cámara experimental permaneció oscurecida y las respuestas emitidas no tuvieron consecuencias programadas. Cada sesión concluyó al completarse 60 ensayos, en los que se presentaron de forma aleatoria las cuatro combinaciones de estímulo posibles. Esta fase estuvo vigente hasta que los sujetos alcanzaron o superaron un índice de precisión de 0.80 durante tres sesiones consecutivas.

Igualación de la muestra demorada: El procedimiento vigente en esta fase fue similar al descrito para la fase anterior, excepto porque se introdujeron IDs de 2, 4 y $8 \mathrm{~s}$ entre la terminación de los EMs y la presentación de los ECOs. Cada valor del ID estuvo vigente durante cinco sesiones consecutivas, en ese orden. La cámara experimental y la tecla de respuesta estuvieron apagadas durante los IDs, y las respuestas emitidas en este periodo no tuvieron consecuencias programadas. 


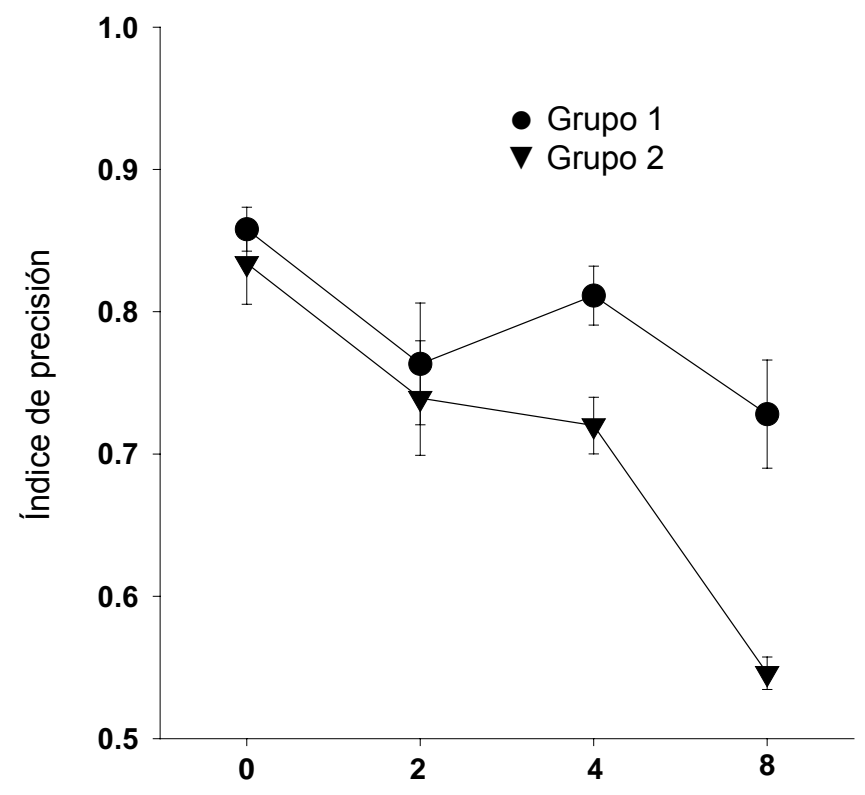

Valor del intervalo de demora (segundos)

Figura 1. Índice de precisión para los grupos 1 (círculos) y 2 (triángulos) durante las últimas 20 sesiones del experimento. Las barras representan los errores estándar de la media.

\section{RESULTADOS}

En promedio, el Grupo 1 requirió de 22.66 sesiones para satisfacer el criterio de estabilidad en la primera fase del experimento, mientras el Grupo 2 requirió 24.33 sesiones. Un análisis estadístico mostró que no existieron diferencias significativas en el número de sesiones requeridas entre ambos grupos $[\mathrm{t}(4)=0.21, \mathrm{p}>.05]$.

La Figura 1 muestra el índice de precisión para el Grupo 1 (círculos) y el Grupo 2 (triángulos), durante las últimas cinco sesiones de la fase igualación de la muestra demora cero y las

15 sesiones de la fase igualación de la muestra demorada, en la que la duración del ID se incrementó a 2, 4 y 8 s. El índice de precisión se calculó a partir de la suma de aciertos por comisión y omisión, dividida entre la suma de estos más los errores por comisión y omisión. En la figura se observa que para el Grupo 2, el índice de precisión disminuyó conforme se incrementó 


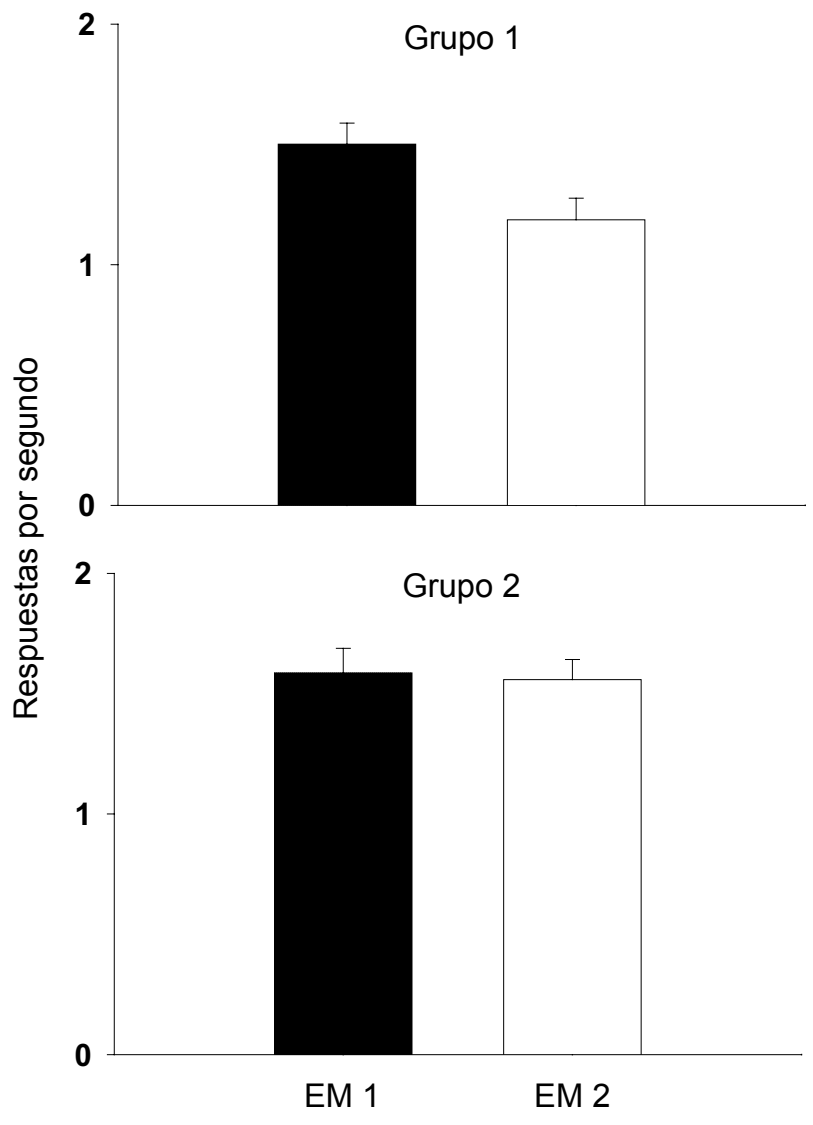

Figura 2. Tasa de respuesta local ante EM1 y EM2 para los grupos 1 (panel superior) y 2 (panel inferior) durante las últimas 20 sesiones del experimento. Las barras representan los errores estándar de la media.

la duración del ID. Para el Grupo 1, incrementar la duración del ID también disminuyó el índice de precisión; sin embargo, para este grupo el efecto fue menor que para el Grupo 2, con un pequeño incremento en el índice de precisión en el valor de $4 \mathrm{~s}$ del ID. Un ANOVA mixto confirmó efectos significativos del IEE $[F(1,28)=12.87, p<.05]$ y la duración del ID $[F(3,28)=18.13, p<$ .05], así como una interacción estadísticamente significativa entre el IEE y la duración del ID $[F(3,28)=3.42, p<.05)]$.

La Figura 2 muestra la tasa de respuesta local ante cada EM para el Grupo 1 (panel superior) y para el Grupo 2 (panel inferior), durante las últimas 20 
Tabla 1. Índices de precisión y tasas de respuesta ante EM1 y EM2 para cada sujeto (promedio por frase).

\begin{tabular}{|c|c|c|c|c|c|c|c|c|c|c|c|c|}
\hline Sujeto & \multicolumn{12}{|c|}{ Demora } \\
\hline & \multicolumn{3}{|c|}{$0 \mathrm{~s}$} & \multicolumn{3}{|c|}{$2 \mathrm{~s}$} & \multicolumn{3}{|c|}{$4 \mathrm{~s}$} & \multicolumn{3}{|c|}{$8 \mathrm{~s}$} \\
\hline & Indice & EM1 & EM2 & Indice & EM1 & EM2 & Indice & EM1 & EM2 & Indice & EM1 & EM2 \\
\hline 1 & 0.83 & $\overline{1.17}$ & 1.27 & 0.63 & $\overline{1.14}$ & $\overline{1.03}$ & 0.79 & $\overline{1.19}$ & $\overline{0.66}$ & 0.70 & $\overline{0.94}$ & $\overline{0.43}$ \\
\hline 2 & 0.87 & 1.52 & 1.10 & 0.81 & 1.50 & 1.03 & 0.77 & 1.21 & 0.66 & 0.76 & 0.79 & 0.26 \\
\hline 3 & 0.86 & 2.28 & 1.97 & 0.83 & 2.41 & 1.93 & 0.86 & 2.06 & 1.97 & 0.72 & 1.75 & 1.90 \\
\hline 4 & 0.79 & 1.20 & 1.61 & 0.69 & 1.46 & 1.52 & 0.69 & 0.77 & 0.91 & 0.55 & 0.66 & 0.96 \\
\hline 5 & 0.86 & 2.13 & 2.16 & 0.83 & 2.25 & 2.26 & 0.72 & 1.66 & 1.91 & 0.53 & 0.90 & 0.78 \\
\hline 6 & 0.85 & 2.46 & 1.85 & 0.68 & 2.63 & 1.91 & 0.74 & 2.14 & 1.95 & 0.54 & 0.74 & 0.83 \\
\hline
\end{tabular}

sesiones del experimento. La figura muestra que para ambos grupos el EM1 controló una tasa de respuesta más elevada que el EM2; sin embargo, esta diferencia entre las tasas de respuesta ante los EMs fue más robusta para el Grupo 1 que para el Grupo 2. Para el Grupo 1, la tasa de respuesta ante el EM1 fue de 1.50 respuestas por segundo, mientras la tasa de respuesta ante el EM2 fue de 1.19 respuestas por segundo. Para el Grupo 2, la tasa de respuesta ante el EM1 fue de 1.59 respuestas por segundo, mientras la tasa de respuesta ante el EM2 fue de 1.56 respuestas por segundo. Un análisis estadístico confirmó que la tasa de respuesta ante los EMs difirió para el Grupo $1[\mathrm{t}(118)=2.51, \mathrm{p}<$. 05] pero no para el Grupo 2 [t(118) $=2.51, \mathrm{p}>.05]$. Un segundo análisis, mostró que la tasa de respuesta global ante los EMs no difirió entre los grupos $[\mathrm{t}(118)=1.83, \mathrm{p}>.05]$.

La Tabla 1 muestra los índices de precisión y tasas de respuesta locales ante cada EM para cada sujeto. Los datos son el promedio de las últimas cinco sesiones de la fase igualación de la muestra demora cero y las cinco sesiones de cada una de las fases experimentales restantes. En general, la tabla confirma los datos grupales. Para los sujetos del Grupo 1, incrementar el valor del ID de 0 a $2 \mathrm{~s}$ disminuyó severamente el índice de precisión sólo para S1, mientras para los otros dos sujetos permaneció por arriba de 0.80 . En el valor de $4 \mathrm{~s}$ del ID, el índice de precisión incrementó respecto de la fase anterior para S1 y S3, mientras para S2 disminuyó ligeramente. En el valor de $8 \mathrm{~s}$ del ID, los índices de precisión disminuyeron para los tres sujetos respecto de la fase anterior, sin embargo, fueron iguales o superiores a 0.70 . Para los sujetos del Grupo 2, incrementar el valor del ID de 0 a 2 s disminuyó severamente los índices de precisión para S4 y S6, mientras para S5 el índice disminuyó ligeramente. En el valor de $4 \mathrm{~s}$ del ID, S5 mantuvo el mismo índice de precisión que en la fase anterior, mientras para S5 el índice de precisión disminuyó nuevamente. Sólo para $\mathrm{S} 6$ el índice de precisión aumentó ligeramente respecto de la fase anterior. En el valor de $8 \mathrm{~s}$ del ID, los índices de precisión disminuyeron para los tres sujetos respecto de la fase anterior, siendo iguales o menores a 0.55 . 
En lo que a la tasa de respuesta ante cada EM se refiere, la Tabla 2 muestra que, con excepción de S1 y S3 en los valores de 0 y 8 s del ID respectivamente, para los sujetos del Grupo 1 la tasa de respuesta fue consistentemente más elevada ante el EM1 que ante el EM2. Para los sujetos del Grupo 2, para S4 la tasa de respuesta fue más elevada ante el EM2 que ante el EM1. Para S5 y S6 se observaron consistencias similares de las tasas de respuesta, pero inversas entre ambos sujetos, en la mayoría de las fases experimentales. La excepción fue en el valor de $8 \mathrm{~s}$ del ID, en el que las tasas de respuesta locales se invirtieron para ambos sujetos. Las diferencias en el responder, sin embargo, fueron menores que para los sujetos del Grupo 1 en la mayoría de las fases experimentales.

\section{DISCUSIÓN}

El presente experimento evaluó los efectos de correlacionar los EMs con IEEs de distinta duración, sobre el índice de precisión en un procedimiento sucesivo de igualación de la muestra demorada. Los resultados mostraron que tanto para el grupo expuesto a la condición correlacionada como para el grupo expuesto a la condición no correlacionada, el índice de precisión disminuyó conforme se incrementó la duración del ID; sin embargo, se observó que el efecto fue menor para el grupo expuesto a la condición correlacionada que para el grupo expuesto a la condición no correlacionada. Adicionalmente, se observó que sólo para el grupo expuesto a la condición correlacionada las tasas de respuesta locales ante los EMs difirieron significativamente.

Los resultados del presente experimento concuerdan con los reportados en estudios previos, en los que la diferenciación de los arreglos de estímulo se realizó mediante la manipulación de otros parámetros del procedimiento de igualación de la muestra demorada. Por ejemplo, Hartl et al. (1996, Experimento 2) y

Urcuioli et al. (1999, Experimento 1), mostraron que correlacionar cada EM con duraciones de presentación específicas, produjo un mayor porcentaje de respuestas correctas respecto de cuando la duración de los EMs se determinó en forma aleatoria. En otro estudio, DeLong y Wasserman (1981, Experimento 1) observaron índices de discriminación más elevados al correlacionar cada EM con una probabilidad de reforzamiento particular, en comparación de cuando la probabilidad de reforzamiento correlacionada con ambos EMs era equivalente.

En relación con las diferencias entre las tasas de respuesta locales ante los EMs, los resultados del presente estudio igualmente concuerdan con los reportados en algunos de los experimentos anteriores. Por ejemplo, tanto en el estudio realizado por Urcuioli et al. (1999, Experimento 1) como en el de 
DeLong y Wasserman (1981, Experimento 1), se observó que la tasa de respuesta local ante los EMs difirió significativamente cuando, respectivamente, cada EM se correlacionó con una duración o probabilidad de reforzamiento particulares. Al respecto, cabe mencionar que otros estudios también han reportado un mayor porcentaje de respuestas correctas al diferenciar los arreglos de estímulo del procedimiento de igualación de la muestra demorada, mediante la correlación de los EMs con diferentes requisitos de respuesta para la presentación de los ECOs (e.g., Cohen, Brady \& Lowry, 1981; Zentall, Hogan, Howard \& More, 1978).

Aunque es posible suponer que diferenciar los arreglos de estímulo en procedimientos de igualación de la muestra demorada tiene efectos similares por la manipulación de uno u otro parámetro, las explicaciones ofrecidas en cada caso no son las mismas. Por ejemplo, de acuerdo con Hartl et al. (1996) sus resultados son evidencia de que la precisión de la ejecución en procedimientos de igualación de la muestra demorada, está determinada por las propiedades reforzantes condicionadas de los

EMs. Desde esta perspectiva, se estima que mientras mayor sea el valor de reducción de demora (Fantino, 1977) señalado por el inicio de un EM, mayor será el porcentaje de respuestas correctas en ese tipo de ensayo (véase Wixted, 1989). Por el otro lado, al igual que en la mayoría de los estudios sobre consecuencias diferenciales (véase Goeters, Blackely \& Poling, 1992), en el caso del experimento conducido por DeLong y Wasserman (1981) se consideró que los índices de discriminación más elevados observados para el grupo expuesto a la condición correlacionada, eran el resultado del desarrollo de expectativas de reforzamiento específicas a cada EM. Desde esta perspectiva, se estima que dichas expectativas de reforzamiento constituyen claves adicionales a los EMs, las cuales guían el responder del organismo en la fase de elección (Trapold \& Overmier, 1972).

Tanto la teoría del valor de reducción de demora (Wixted,1989) como la de expectativas de reforzamiento (Trapold \& Overmier, 1972), constituyen claros ejemplos de lo que Cabrer, Daza y Ribes (1975) denominaron enfoques centrados en el problema, es decir, estrategias analítico-explicativas que introducen nuevos modelos y conceptos para dar cuenta de aparentes fenómenos experimentales. Dado que tales fenómenos por lo general constituyen el efecto de la manipulación implícita de variables no identificadas, dichos autores propusieron desechar el enfoque centrado en el problema y privilegiar el enfoque paramétrico, es decir, el análisis sistemático de las características de los distintos procedimientos, con el fin de encontrar en ellos parámetros comunes que permitan integrar en un solo marco descriptivo los aparentes fenómenos experimentales.

Desde nuestro punto de vista, los resultados del presente experimento demuestran las limitaciones del enfoque centrado en el problema y confirman 
en valor heurístico del enfoque paramétrico. Por ejemplo, aunque en el presente estudio no se analizó el índice de precisión por tipo de ensayo, el valor de reducción de demora promedio señalado por los EMs tanto en la condición correlacionada como en la no correlacionada, era el mismo. No obstante, el índice de precisión fue mayor para el grupo expuesto a la condición correlacionada que para el grupo expuesto a la condición no correlacionada. Por el otro lado, resulta imposible suponer que para los sujetos expuestos a la condición correlacionada se desarrollaran expectativas de reforzamiento específicas a cada EM, simplemente porque en el presente estudio el tipo de reforzador correlacionado con ambos estímulos era el mismo.

En contraste con las posiciones anteriores, desde un punto de vista paramétrico los resultados del presente experimento, confirman la continuidad de los efectos del IEE entre los procedimientos de igualación de la muestra demorada y los de automoldeamiento, sugerida por Roberts y Kraemer (1982). No obstante, a diferencia de lo propuesto por estos autores, nuestros resultados dejan ver que los efectos del IEE no se limitan a su valor promedio, sino que se extienden a sus valores locales por tipo de ensayo. En el presente experimento, el IEE promedio fue equivalente entre los procedimientos de la condición correlacionada y la no correlacionada. En línea con esta característica, no se encontraron diferencias en la velocidad de adquisición de la discriminación condicional en la fase de igualación de la muestra demora cero, ni tampoco entre las tasas de respuesta globales ante los EMs de ambos grupos. No obstante, para el grupo expuesto a la condición correlacionada la tasa de respuesta local fue más elevada ante el EM que se correlacionó con el IEE más largo (e.g., Perkins, et al., 1975, Experimento 5). Al parecer, y en línea con los resultados de varios estudios previos (e.g., Alling, Nickel \& Poling, 1991; Brodigan \& Peterson, 1976; Cohen, Brady \& Lowry, 1981; Zentall, Hogan, Howard \& More, 1978; Lattal, 1975), fueron precisamente estas diferencias en el responder ante los EMs las que favorecieron la precisión de la ejecución. Las menores diferencias de tasa local observadas para dos de los sujetos del Grupo 2, aunadas a las diferencias de tasa local y el incremento en la precisión observados para S6, igualmente apoyan este planteamiento.

La continuidad de los efectos del IEE antes aludida, sin embargo, no significa que la configuración del comportamiento sea equivalente en los procedimientos de automoldeamiento e igualación de la muestra demorada. De hecho, mientras en los procedimientos de automoldeamiento la distribución de respuestas simplemente se ajusta a la distribución temporal de los estímulos, en los procedimientos de igualación de la muestra la distribución temporal de los estímulos está regulada por otros estímulos y las respuestas precisas del organismo respecto de la distribución temporal de estos últimos (Carpio, 1994). La continuidad se refiere, más bien, a que los efectos de los parámetros involucrados en la configuración del comportamiento en el primer 
caso, están incluidos -aunque de manera subordinada- en la configuración del comportamiento en el segundo caso (Ribes \& López, 1985). Los resultados del presente experimento proporcionan evidencia favorable a este planteamiento. Futuros estudios deberán determinar si diferenciar los arreglos de estímulo mediante la manipulación de uno u otro parámetro de los procedimientos de igualación de la muestra demorada, produce diferencias en las tasas de respuesta locales ante los EMs.

\section{REFERENCIAS}

Alling, K., Nickel, M., \& Poling, A. (1991). The effects of differential and no differential outcomes on response rates and accuracy under a delayed-matching-to-sample procedure. The Psychological Record. 41, 537-549.

Berryman, R., Cumming, W. W., \& Nevin, J. A. (1963). Acquisition of delayed matching in the pigeon. Journal of the Experimental Analysis of Behavior. 6, 101-107.

Brodigan, D. L., \& Peterson, G. B. (1976). Two-choice conditional discrimination performance of pigeons as a function of reward expectancy, prechoice delay, and domesticity. Animal Learning \& Behavior. 4, 121-124.

Brown, P. L., \& Jenkins, H. M. (1968). Auto-shaping of the pigeon's key-peck. Journal of the Experimental Analysis of Behavior. 11, 1-8.

Cabrer, F., Daza, C., \& Ribes, E. (1975). Teoría de la conducta: ¿Nuevos conceptos o nuevos parámetros? Revista Mexicana de Análisis de la Conducta. 1, 191-212.

Carpio, C. (1994). Comportamiento animal y teoría de la conducta. En L. J. Hayes, E. Ribes \& F. López (Eds.), Psicología interconductual: Contribuciones en honor a J. R. Kantor (pp. 45-68). México: Universidad de Guadalajara.

Cohen, L. R., Brady, J., \& Lowry, M. (1981). The role of differential responding in matching-to-sample and delayed matching performance. En M. L. Commons, \& J. A. Nevin (Eds.), Quantitative analysis of behavior: Discrimination proprieties of reinforcement schedules (pp. 345-364). Cambridge, Massachusetts: Ballinger Publishing Company.

DeLong, R. E., \& Wasserman, E. A. (1981). Effects of differential reinforcement expectancies on successive matching-to-sample performance in pigeons. Journal of Experimental Psychology: Animal Behavior Processes. 7,394-412.

Fantino, E. (1977). Conditioned reinforcement: Choice and information. En W. K. Honig \& J. E. R. Staddon (Eds.), Handbook of operant behavior (pp. 313-339). Englewood Cliffs, NJ: Prentice Hall.

Goeters, S., Blakely, E., \& Poling, A. (1992). The differential outcomes effect. The Psychological Record. 42, 389-411.

Hartl, J. A., Dougherty, D. H., \& Wixted, J. T. (1996). Separating the effects of trialspecific and average sample-stimulus duration in delayed matching to sample in pigeons. Journal of the Experimental Analysis of Behavior. 66, 231-242.

Honig, W. K. (1987). Memory interval distribution effects in pigeons. Animal Learning \& Behavior. 15, 6-14. 
Lattal, K. A. (1975). Reinforcement contingencies as discriminative stimuli. Journal of the Experimental Analysis of Behavior. 23, 241-246.

Nelson, K. R., \& Wasserman, E. A. (1978). Temporal factors influencing the pigeon's successive matching-to-sample performance: Sample duration, intertrial interval, and retention interval. Journal of the Experimental Analysis of Behavior. 30, 15362.

Perkins, C. C., Beavers, W. O., Hancock, R. A., Hemmendinger, P. C., Hemmendinger, D., \& Ricci, J. A. (1975). Some variables affecting rate of key pecking during response-independent procedures (autoshaping). Journal of the Experimental Analysis of Behavior. 24, 59-72.

Ribes, E., \& López, F. (1985). Teoría de la conducta: Un análisis de campo y paramétrico. México: Trillas.

Roberts, W. A., \& Kraemer, P. J. (1982). Some observations of the effects of intertrial interval and delay on delayed matching to sample in pigeons. Journal of Experimental Psychology: Animal Behavior Processes. 8, 342-353.

Trapold, M. A., \& Overmier, J. B. (1972). The second learning process in instrumental learning. En A. A. Black, \& W. F. Prokasy (Eds.), Classical conditioning: Vol. 2. Current research and theory (pp. 427-452). New York: Appleton Century Crofts.

Urcuioli, P. J., DeMarse, T. B., \& Lionello, K. M. (1999). Sample-duration effects on pigeons' delayed matching as a function of predictability of duration. Journal of the Experimental Analysis of Behavior. 72, 279-297.

Wixted, J. T. (1989). Nonhuman short-term memory: A quantitative reanalysis of selected findings. Journal the Experimental Analysis of Behavior. 52, 409-426.

Zentall, T. R., Hogan, D. E., Howard, M. M., \& Moore, B. S. (1978). Delayed matching in the pigeon: Effect on performance of sample-specific observing responses and differential delay behavior. Learning and Motivation. 9, 202-218. 